\title{
An Operation - Reduced Calculation Method for Solving Complex Communication Systems
}

\author{
Vladimir MLADENOVIC ${ }^{1}$, Danijela MILOSEVIC ${ }^{2}$, Miroslav LUTOVAC ${ }^{3}$ \\ ${ }^{1}$ University of Kragujevac, Faculty of Technical Sciences in Cacak, Svetog Save 65, Cacak, 32000, Serbia \\ vladimir.mladenovic@ftn.kg.ac.rs \\ ${ }^{2}$ University of Kragujevac, Faculty of Technical Sciences in Cacak, Svetog Save 65, Cacak, 32000, Serbia \\ danijela.milosevic@ftn.kg.ac.rs \\ ${ }^{3}$ Singidunum University, Belgrade, Danijelova 32, Belgrade, 11000, Serbia \\ lutovac@gmail.com
}

\begin{abstract}
Calculation and design of complex communication systems are usually based on expert knowledge in telecommunications and through the usage of numeric software tools. According to various requests and initial conditions, the software procedure can be time consuming due to a large number of operations. This paper incorporates a new approach focused on a novel concept of calculation in optimisation procedures of complex communication systems, which reduces the number of operations. The key point is defining an auxiliary function and modelling a new optimised function suitable for further manipulation and calculation. We evaluate the hypothesis that a fast yet accurate approximation of an error probability is possible using a parametric function. It is used for calculation and analysis of error probability using an optimised function. The method is verified and validated on PC and Raspberry pi.
\end{abstract}

Keywords: Closed-form solution, Wolfram language, Rayleigh fading, Software tools, Computer algebra system

\section{Introduction}

It is well known that researchers and engineers use more with methods in calculations of systems and processes when compared to traditional information delivery through the presentation. This is especially obvious for the upcoming technologies in the field of telecommunications, such as $5 \mathrm{G}$ networks. Their rapid development and standardisation bring a large number of complex techniques to transfer information [16]. Also, the modern 5G communication systems require near-real time estimation of error probability [1].

The complexity of the system will be reflected in an increase in the number of customers, services increasing and a comprehensive integration of individual "smart" systems. It will be especially interesting challenges to the saving of the frequency space, device-todevice channels, fixed-to-vehicle channels, mobile-to-mobile communications, vehicle-tovehicle channels, vehicular communications, moving scatterers [2], [12]. This will result needs for finding various algorithms and methodologies to speed up the calculation as an integral step in the design of these systems. On the other side, the calculations during design necessity use of mathematical integrals include iterative processes to get error probability [3]. In this sense, the paper presents a method that contributes to the calculation tools of complex techniques in these networks called the operation-reduced calculation method (ORCM). The presented method helps engineers and researchers to accelerate their calculations by reducing the number of operations. It will greatly help with a numerical calculation that can be considered as a link between theoretical and experimental analysis. For efficiently solving the optimal result is an original procedure developed using Wolfram language [18]. The main motivation for the development and application of this algorithm is the question whether a system exists that must be extremely fast and accurate enough to calculate error probability. Such a response is provided in this paper.

This paper is structured as follows. In section 2, the current state is presented from viewpoint of kind of computation and subject of researches. In section 3, knowledge programming using the computer algebra system and Wolfram language is introduced. Section 4 describes the problem overview and algorithm formulation. In section 5, the code-deriving closed-form solutions of parameters of probability by using an operation - reduced calculation method and calculations, analysis and results are illustrated in section 6 . 


\section{Related Work}

In the last decade, many new algorithms have been created to speed up calculating, improve and solve performances. They provide results with parameters have not been taken in observation, but significantly influence to the design of wireless systems as in [14].

The growth of urban areas with tall buildings with significant density and a sharp rise in users of mobile communications lead to the finding of useful methods and ways for proper data exchange. It starts from the viewpoint of data exchange, and the parameters needed for calculation of the proper functioning of the system under all conditions of design rules in mobile systems [11], [12]. The analyses and calculations of the parameters usually perform statistical observations using by numericalbased tools as in [7]. Often, it can represent a bottleneck of very complex analysis in cases when analytical expressions are needed, which describe the observed system. However, various kinds of investigation are used for an analysis of the performance of wireless systems for different scenarios. The most common form of calculations is based on traditional numerical tools as in [2,], [4], [5], [13].

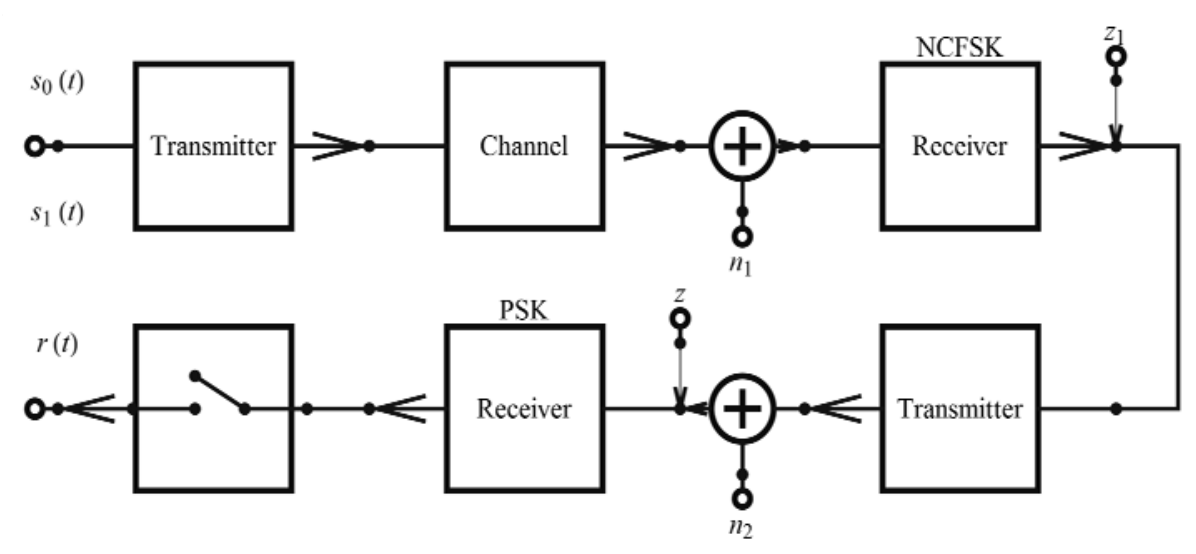

Figure 1. Complex NCFSK/PSK communication system.

\section{Computer Algebra System and Wolfram Language}

The Wolfram language (WL) is the programming language that is suitable as computer algebra system (CAS) with an ability to manipulate symbolic expressions in a way similar to traditional manual derivation. The exceptional property of WL is the highperformance rule-based computing so that program codes can be generated in a short and compact form. It supports also object-oriented, procedural, parallel, and functional programming. Specific application packages can be produced using development environments (Eclipse and Wolfram Workbench), such as SchematicSolver [6]. Large number of notebook documents as template documents contain program code, text, figures, tables and dynamic visualisation of algorithms. Powerful features for solving and simulating continuous, discrete-time, and nonlinear system were reported in number of journal papers [8], [9]. Wolfram language is also available on cheap Raspberry Pi hardware environment with additional general-purpose input/output (GPIO) programming and serial communication that are of great importance for internet of things applications.

\section{The Problem Formulation and Algorithm Overview}

The ORCM is illustrated on the calculation of complex NCFSK/PSK (non-coherent frequency-shift keying/phase-shift keying) wireless communication system shown in Figure 1. Similar part of the system, where real-time estimation is needed, can be found in [15]. This system consists of two cascade stages of different digital modulation techniques. The one is FSK (marked as NCFSK receiver block), and second is PSK (marked as $P S K$ receiver block). The prefix of NC is used for non-coherent detection in the first stage. 
The output of the first stage is the input of the second stage, and the signal between two stages is marked as $z_{1}$, in following described as part of the expression (3), more precision $z_{1}=z_{0 \mathrm{i}}+z_{1 \mathrm{i}}$ $(i=0,1)$. The last block, with the $r(t)$ output, is sampler for decision of received information. The mentioned system is analysed using the following steps:

1. defining of hypotheses and distributions. Assumption is to exist one dominant signal and multipath, so, the consequence is Rician distribution;

2. transformation of the expressions and calculation the probability distribution function of errors probability using an iterative-reduced simulation method [9], [10];

3. defining the approximate function and calculate the coefficients;

4. calculation of the error function between the exact value of the error probability and the approximate function;

5. calculation of the optimal number of operations.

The ORCM starts with the exact value of the observed function. We define the approximate function that is the most similar to the original function, but with different mathematical description. Approximated function includes coefficients that are calculated on the basis of a system of equations. According to this, we derive the optimal number of operations, error function, and new error probability.

The error function is checked using relative error. Let us run the practical application on the observed system. The input signal $s_{\mathrm{i}}(t)(i=0,1)$ is present on input of system with hypothesis

$$
\begin{aligned}
& H_{0}: s_{0}(t)=A \cos \left(\omega_{0} t\right) \\
& H_{1}: s_{1}(t)=A \cos \left(\omega_{1} t\right)
\end{aligned}
$$

Informations pass through the wireless channel with Rician fading and narrowband noise $n_{1}(t)$, marked as Channel block in Figure 1, and come to the first stage of the NCFSK receiver. The NCFSK is composed by two branches with two different frequencies. The first one is valid for the signal $s_{0}(t)$ and the second branch is valid for the signal $s_{1}(t)$ with envelops

$$
\left.\mathrm{z}_{\mathrm{ij}}\right|_{(\mathrm{i}, \mathrm{j}) \in(0,1)}=\left\{\begin{array}{l}
\sqrt{\left(A+x_{1}\right)^{2}+\left(A+y_{1}\right)^{2}}, \text { for } i=j \\
\sqrt{x_{1}{ }^{2}+y_{1}{ }^{2}}, \text { for } i \neq j
\end{array}\right.
$$

So, the envelops $z$ are

$$
z=\left(z_{0 i}+z_{1 i}\right)+n_{2},(i=0,1)
$$

for hypothesis $H_{0}$ and $H_{1}$, and $n_{2}$ is noise in the second stage of the system. Probability density functions of signal at the output of the first stage are

$$
\left.\mathrm{p}\left(\mathrm{z}_{\mathrm{ij}}\right)\right|_{(\mathrm{i}, \mathrm{j}) \in(0,1)}=\left\{\begin{array}{l}
\frac{\mathrm{z}_{\mathrm{ij}}}{\sigma_{1}^{2}} \cdot e^{-\frac{z_{i j}^{2}+A^{2}}{2 \sigma_{1}^{2}}} \cdot \mathrm{I}_{0}\left(\frac{z_{i j} \cdot A}{\sigma_{1}^{2}}\right), \text { for } i=j \\
\frac{\mathrm{z}_{\mathrm{ij}}}{\sigma_{1}^{2}} \cdot e^{-\frac{z_{i j}^{2}}{2 \sigma_{1}^{2}}}, \text { for } i \neq j
\end{array}\right.
$$

where $I_{0}(\cdot)$ is Bessel function zero order.

Distribution functions of the envelops in the second branch for hypothesis $H_{0}$ and $H_{1}$ are

$$
\begin{aligned}
\left.p_{i}(r)\right|_{i=0,1} & =\int_{z_{0 i}} \int_{1_{1 i}} \frac{r}{\sigma_{2}^{2}} e^{-\frac{r^{2}+\left(z_{0 i}-z_{1 i}\right)^{2}}{2 \sigma_{2}{ }^{2}}} \times \\
& \times I_{0}\left(\frac{\left(z_{0 i}-z_{1 i}\right) \cdot r}{\sigma_{2}^{2}}\right) \cdot p\left(z_{0 i}\right) p\left(z_{1 i}\right) d z_{0 i} d z_{1 i}
\end{aligned}
$$

here $0 \leq\left(z_{0 \mathrm{i}}, z_{1 \mathrm{i}}\right) \leq \infty$. Taking into consideration the presence of Rician distribution, the $r$ presents the envelop of received signal. Finally, the total error probability is:

$$
P_{e}=\frac{1}{2}\left\{\int_{0}^{\infty} p_{0}(r) d r+\int_{0}^{\infty} p_{1}(r) d r\right\}
$$

The error probability function is often calculated using by suitable numerical algorithms that involve iteration steps. A large number of iterations follows a large number of operations during calculation, so, it can take a time-consuming [8]. We use similar iterative method as in [9], [10] to get error probability function. In the following, we use the label $P_{e}$ for the original total error probability, and $P_{\text {e,approx }}$ for the approximative total error probability. For approximative function we define

$$
P_{e, \text { approx }}=a_{1} \cdot \operatorname{erfc}\left(b_{1} \cdot A+b_{2}\right)+a_{2}
$$

where $a_{1}, a_{2}, b_{1}, b_{2}$ are coefficients, the $A$ is the envelop, and $\operatorname{erfc}(\cdot)$ is the complementary error function defined by

$$
\operatorname{erfc}(x)=\frac{2}{\sqrt{\pi}} \int_{x}^{\infty} e^{-t^{2}} d t
$$

The relative error function is obtained using standard definition as

$\delta=\frac{P_{e, \text { approx }}}{P_{e}}-1$ 


\section{Code-deriving closed-form solutions of parameters of probability by using an operation - reduced calculation method}

The code writing mains the generating of statements in the same way as we write a mathematical expression. This is simply named as the natural language programming. In that sense, the realization of the operation-reduced calculation method procedure is presented with the same steps as in previous sections. So, the distribution functions are written according to the expression (4) as:

$$
\begin{aligned}
& \text { pzo0 }\left[z 00_{-}, s 1_{-}, A_{-}\right]:= \\
& \frac{z 00}{(s 1)^{2}} \star \operatorname{Exp}\left[-\frac{(z 00)^{2}+A^{2}}{2 *(s 1)^{2}}\right] \star \operatorname{Bessel}\left[0, \frac{z 00 * A}{(s 1)^{2}}\right] ; \\
& p z 10\left[z 10_{-}, s 1_{-}, A_{-}\right]:=\frac{z 10}{(s 1)^{2}} \star \operatorname{Exp}\left[-\frac{(z 10)^{2}}{2 *(s 1)^{2}}\right] ; \\
& p z 01\left[z 01_{-}, s 1_{-}, A_{-}\right]:=\frac{z 01}{(s 1)^{2}} * \operatorname{Exp}\left[-\frac{(z 01)^{2}}{2 *(s 1)^{2}}\right] ; \\
& p z 11\left[z 11_{-}, s 1_{-}, A_{-}\right]:= \\
& \frac{z 11}{(s 1)^{2}} * \operatorname{Exp}\left[-\frac{(z 11)^{2}+A^{2}}{2 *(s 1)^{2}}\right] * \operatorname{Bessel}\left[0, \frac{z 11 * A}{(s 1)^{2}}\right] ;
\end{aligned}
$$

Figure 2. Distribution functions written in Wolfram language

The marks $z 0 \odot, z 01, z 10$ and $z 11$ present $z_{i j}$. The mark BesselI $[\odot, \bullet]$ is $I_{0}(\cdot)$, and s1 is $\sigma_{1}$. The operator := means assigning one variable or more variables to function with delayed values.

Distribution functions defined in expression (5) are realised over RiemannSumDouble function introduced in [10], and encapsulated into code as:

$$
\begin{aligned}
& \operatorname{pr} 1\left[r_{-}, q_{-}, A_{-}, s 1_{-}, s 2_{-}\right]:= \\
& \text {RiemannSumDouble }\left[\frac{r}{(s 2)^{2}} * \operatorname{Exp}\left[-\frac{r^{2}+(z 01-z 11)^{2}}{2 *(s 2)^{2}}\right]\right. \\
& \star \text { BesselI }\left[0, \frac{(z 01-z 11) * r}{(s 2)^{2}}\right] * \operatorname{pz} 01[z 01, s 1, A] * \\
& \quad \operatorname{pz} 11[z 11, s 1, A],\{z 01,0,1\},\{z 11,0,1\}, q]
\end{aligned}
$$

Figure 3. Complete distribution functions for hypothesis $H_{0}$ and $H_{1}$

where the mark s2 is $\sigma_{2}$, the $r$ is the envelop, the $q$ is the iteration step, and boundaries are normalised in integral. Similarly, the single Riemann sum is defined as follows [6]:

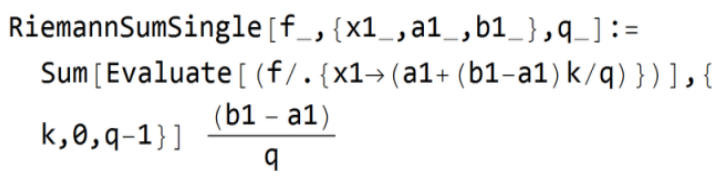

Figure 4. Single Riemann sum defined using Wolfram language

The last code in figure 4 is used to define the total error probability in expression (6):

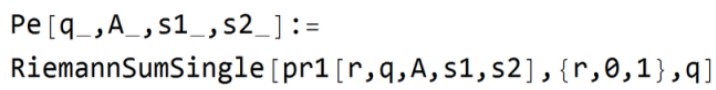

Figure 5. The total error probability in Wolfram language

Now, we define the approximative total error probability as in (7):

fun $\left[\mathrm{a} 1_{-}, \mathrm{n} 1_{-}, \mathrm{a} 2_{-}, \mathrm{n} 2_{-}, \mathrm{A}_{-}\right]:=\mathrm{a} 1 * \operatorname{Erfc}[\mathrm{A} * \mathrm{n} 1+\mathrm{n} 2]+\mathrm{a} 2$

Figure 6. Defining of the approximative total error probability in Wolfram language

Calculation of coefficients the $a_{1}, b_{1}, a_{2}$ and $b_{2}$ is performed by equalising the exact value in $100^{\text {th }}$ iteration from Figure 5 with four setpoints in Figure 6. So,

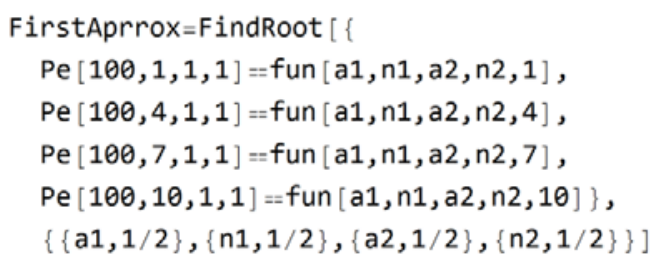

Figure 7. Calculation of coefficients of approximative total error probability in four setpoints

The command FindRoot searching of roots of defined function $f$, starting from the set points. In our case, we defined the startpoints as $a_{1}=a_{2}=b_{1}=b_{2}=1 / 2$. Obtained coefficients are used in the further procedure for testing of approximative expression through the relative error function according to expression (9) as:

$$
\begin{aligned}
& \text { errorRelatiVe }\left[\mathrm{q}_{-}, \mathrm{A}_{-}, \mathrm{s} 1_{-}, \mathrm{s} 2_{-}, \mathrm{a} 1_{-}, \mathrm{n} 1_{-}, \mathrm{a} 2_{-},\right. \\
& \left.\mathrm{n} 2_{-}\right]:=\frac{\text { fun }[\mathrm{a} 1, \mathrm{n} 1, \mathrm{a} 2, \mathrm{n} 2, \mathrm{~A}]}{\operatorname{Pe}[\mathrm{q}, \mathrm{A}, \mathrm{s} 1, \mathrm{~s} 2]}-1
\end{aligned}
$$

Figure 8. Relative error function in term of the envelop $A$ 


\section{Calculations, Analysis and Results}

The start point is the calculation of coefficients in approximative function. For given $\sigma_{1}$ and $\sigma_{2}\left(\sigma_{1}=1 ; \sigma_{2}=1,2,3\right)$, we determine the parameters of the approximation such that the approximation and the error probability are computed using the expression (6) (coded as in Figure 5) in $100^{\text {th }}$ iterations match for four selected values of the envelop $A$ with 1, 4, 7 and 10. So, the coefficients take values: $a_{1}=\left(5.15 \cdot 10^{-4}, \quad 1.54 \cdot 10^{-4}, \quad 7.06 \cdot 10^{-5}\right) \quad$ and $b_{1}=7.1 \cdot 10^{-1}, a_{2}=0$ and $b_{2}=-6.93 \cdot 10^{-1}$.

The algorithm is tested on Raspberry pi computer with ARM (32-bit) processor (950 $\mathrm{MHz}, 128 \mathrm{MB}$ GPU). The Raspbian v2.1.0

The algorithm is tested on Raspberry pi computer with ARM (32-bit) processor (950 $\mathrm{MHz}, 128 \mathrm{MB}$ GPU). The Raspbian v2.1.0 operating system is installed with Mathematica Wolfram 10.3.1.0. Average time consuming during calculations is $7.775 \mathrm{~s}$. The time consuming for calculation of approximative error probability functions is with order of $10^{-6} \mathrm{~s}$. Wolfram language calculates error probability expression (6) using a sum in Figure 2, 34 and 5 where $N I$ is the number of iterations. So, we obtain the closed-form expression of $P_{e}$ in form:

$P_{e}=\frac{\exp \left(-\frac{A^{2}}{2 \sigma_{1}^{2}}\right)}{(N I)^{5} \sigma_{1}^{4} \sigma_{2}^{2}} \times$

$\times \sum_{k=0}^{N I-1} k^{3} \exp \left(-\frac{k^{2}\left(\sigma_{1}^{2}+\sigma_{1}^{2}\right)}{(N I)^{2} \sigma_{1}^{2} \sigma_{2}^{2}}\right) \cdot I_{0}\left(\frac{A \cdot k}{N I \cdot \sigma_{1}^{2}}\right)$

Closed-form expression (10) is obtained with manipulation and simplification automatically using symbolic tools defined in WL code in Figure 2, 3, 4 and 5, too.

Moreover, Wolfram language successful counts the $N O$ (number of operations) in term of $N I$ in expression (10), such as Times, Power, BesselI, Plus, Rational using command StringPosition. After counting the operations in the expression (10) we obtain the relation between number of operations and number of iterations. So,

$N O=116(N I)^{2}$

Table 1 shows the number of operations for a various number of iterations of $P_{e}$.
Table 1. Increasing the $N O$ of $P_{e}$ in term of $N I$ for $\sigma_{1}=\sigma_{2}=1$

\begin{tabular}{|c|c|}
\hline NI & NO in eq. (10) \\
\hline & \\
\hline 1 & 116 \\
\hline 22 & 56144 \\
\hline 43 & 214484 \\
\hline 64 & 475136 \\
\hline 85 & 838100 \\
\hline
\end{tabular}

Also, we count the number of operations for approximative function in expression (7). The counting is performed using command StringPosition, too. The total number of operations is five operations. But, the number of operations is one less because of $a_{2}=0$.

Figure 9 shows changing of the relative error function, defined in (9), in term of the envelop $A$.

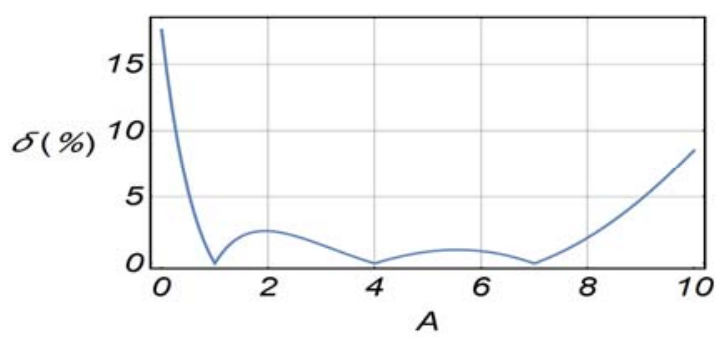

Figure 9. Relative error function in term of the envelop $A$

Figure 10 shows original and approximative error probabilities for given $\sigma_{1}$ and $\sigma_{2}$. It can be seen that the approximative probability error function is very precision according to original error probability.

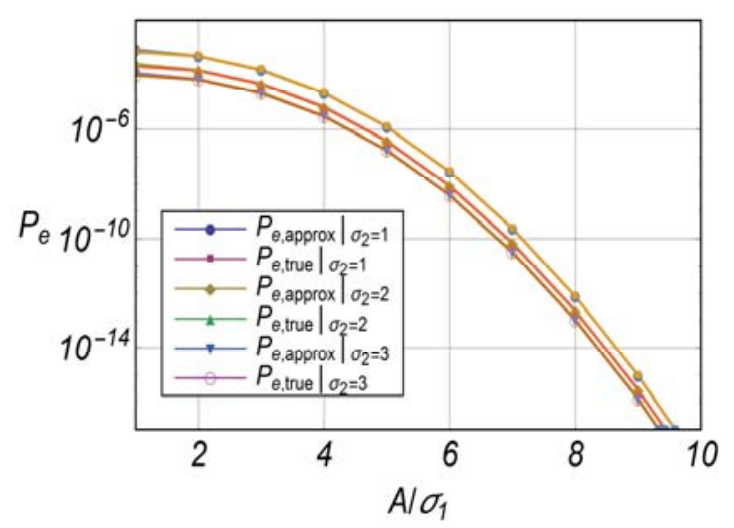

Figure 10. Approximative and original error probability function

\section{Conclusion}

Calculation, analysis and design of complex communication systems are usually based on numeric software tools. Often, these tools are developed with algorithms based on various 
iterative methods. According to various requests and initial conditions, the algorithm's procedures can time consuming due to a large number of operations. In this paper, the novel concept of calculation in optimisation procedures of complex communication systems is introduced that reduces the number of operations. The main point is defining of auxiliary function as an approximative function suitable for further manipulation and calculation. The results are shown through the error function and the counting of the number of operations. The formal verification and testing were done on Raspberry pi computer.

\section{Acknowledgements}

This work was supported by Ministry of Education and Science of Serbia under Grant TR 32023.

Also, the authors thank to prof. David Donald Pokrajac from Delaware State University, Dover, DE, 19901, USA, for suggestions and comments during the writing of this paper.

\section{REFERENCES}

1. Andrews J. G., Buzzi S., Choi W., Hanly S. V., Lozano A., Soong A.C.K. \& Zhang J. C. (2014). What Will 5G Be?, IEEE Journal on Selected Areas in Communications, 32(6), 1065 - 1082.

2. Borhani A. \& Patzold M. (2012). Modelling of Vehicle-to-Vehicle Channels in the Presence of Moving Scatterers, Vehicular Technology Conference, Quebec City, Canada, (pp. 1-5)

3. Devendra K. C. (2009). Modeling and Simulation of Systems Using MATLAB and Simulink, CRC Press.

4. Hajri N., Youssef N. \& Patzold M. (2015). On the Statistical Properties of Phase Crossings and Random FM Noise in Double Rayleigh Fading Channels, IEEE Transactions on Vehicular Technology, 65(4), $1859-1867$.

5. Hogstad B. O., Gutierrez C. A., Patzold M. \& Crespo P. M. (2013). Classes of sum-ofcisoids processes and their statistics for the modeling and simulation of mobile fading channels, EURASIP Journal on Wireless Communications and Networking, 2013(1), 1-15

6. http://www.wolfram.com/products/applicat ions/schematicsolver/manual.pdf
7. Jaksic B., Stefanovic D., Stefanovic M., Spalevic P. \& Milenkovic V. (2015). Level Crossing Rate of Macrodiversity System in the Presence of Multipath Fading and Shadowing, Radioengineering, 24(1), 185-191.

8. Mladenovic V., Lutovac M. \& Porrat D. (2014). Symbolic Analysis as Universal Tool for Deriving Properties of Non-linear Algorithms - Case study of EM Algorithm, Acta Polytechnica Hungarica, 2014, 11(2), 117-136

9. Mladenovic V., Makov S., Voronin V. \& Lutovac M. (2016). An Iteration-Based Simulation Method for Getting SemiSymbolic Solution of Non-coherent FSK/ASK System by Using Computer Algebra Systems, Studies in Informatics and Control, 25(3), 303-312

10. Mladenovic V. \& Milosevic D. (2016). A Novel-Iterative Simulation Method for Performance Analysis of Non-Coherent FSK/ASK Systems Over Rice/Rayleigh Channels using the Wolfram Language, Serbian Journal of Electrical Engineering, 13(2), 157-174

11. Patzold M. (2002). Mobile fading channels, John Wiley \& Sons Ltd, pp. 33.

12. Patzold, M. (2011). Mobile Radio Channels, 2nd ed. Chichester, John Wiley \& Sons.

13. Patzold M., Killat U., Li Y. \& Laue F. (1997). Modeling, Analysis, and Simulation of Nonfrequency-Selective Mobile Radio Channels with Asymmetrical Doppler Power Spectral Density Shapes, IEEE Transactions on Vehicular Technology, 46 (2), 494- 507.

14. Pavlovic V., Mladenovic V. \& Lutovac M. (2014). Telecommunications: Applications, Modern Technologies and Economic Impact, [Book chapter], Nova Science Publishers, New York, USA, pp 29

15. Radwan A., Rodriguez J. (2014). Energy Efficient Smart Phones for $5 G$ Networks, Springer.

16. Rodriguez J. (2015). Fundamentals of $5 G$ Mobile Networks, John Wiley \& Sons, Ltd.

17. Weisstein E. W., Riemann Sum, From MathWorld--A Wolfram Web Resource.

18. Wolfram S. (2015). An Elementary Introduction to the Wolfram Language, Wolfram Media, Inc, Campaign, USA. 